\title{
Dormición y tipo de suelo como determinantes de la germinación y establecimiento de Chloris berroi en la Pampa Deprimida
}

\author{
Víctor A. Bolaños ${ }^{1, \otimes} ;$ M. CRistinaVecchio ${ }^{1} \&$ Rodolfo A. Golluscio ${ }^{2}$ \\ 1. Facultad de Ciencias Agrarias y Forestales. Universidad Nacional de La Plata. 2. Facultad de Agronomía. Universidad de \\ Buenos Aires. IFEVA (UBA- CONICET).
}

\begin{abstract}
Resumen. En los pastizales halófitos de la Pampa Deprimida la historia pastoril habría inducido deterioros en la diversidad florísticos y propiedades edáficas. La recuperación de las especies nativas de alto valor forrajero constituye una valiosa estrategia para la regeneración forrajera del sistema. Ello requiere un profundo conocimiento de los aspectos ecológicos de estas especies que, en el caso de Chloris berroi, nativa y potencial regeneradora de ambientes degradados, es desconocid. En este trabajo evaluamos dos hipótesis: (1) la dormición de $C$. berroi se debe a las estructuras que recubren la cariópside e inhiben su germinación durante un cierto período después de la cosecha y (2) el establecimiento de plántulas de C. berroi es afectado por el tipo de suelo. Realizamos dos ensayos: uno evaluó el efecto de la remoción de glumas y glumelas sobre la germinación en semillas de $C$. berroi con dos tiempos de almacenamiento ( 2 y 8 meses) y sembradas sobre tres suelos distintos (dos Natracualf típicos de estepa de halófitas contiguas pero con diferentes historias en el manejo del pastoreo (continuo/rotativo) y un Argiudol Vértico de una pradera húmeda de mesófitas con pastoreo rotativo). El segundo evaluó la capacidad de establecimiento de la especie sobre cada suelo. La remoción de la cubierta seminal promovió significativamente la germinación solo en las semillas de menor tiempo de almacenamiento. El tipo de suelo no afectó la germinación, en tanto, el establecimiento de plántulas se redujo significativamente sobre el Natracualf bajo pastoreo continuo. Los resultados sugieren que efectivamente las cubiertas seminales inducirían dormición por algunos meses $(<8)$. El pastoreo rotativo favorecería la preservación de $C$ berroi en las comunidades halófitas y su ausencia en las comunidades vegetales correspondiente a suelos Argiudol vértico no respondería a cuestiones edáficas.
\end{abstract}

[Palabras clave: pastizal, ambiente halomorfico, regeneración, especie nativa, tipo de pastoreo]

\begin{abstract}
Aвstract. Dormancy and soil type as determinant for germination and establishment of Chloris berroi in the Flooding Pampa: In halophytic grassland of the Flooding Pampa, domestic grazing might have induced losses in floristic diversity as well as in soil properties. The recovery of native species with high forage value is a valuable strategy for the system regeneration. This requires a thorough understanding of the ecology of the target species, unknown for Chloris berroi, a native and potential regenerative of degraded environments. We tested two hypotheses: (1) C. berroi dormancy is due to the seed covers that inhibit their germination for a certain interval after harvest; and (2) C. berroi plants, during their germination, are affected by soil type. We carried out two essays: the first evaluated the effect that removal glumes exerted on the seed germination of $C$. berroi with two storage periods ( 2 and 8 months) and seeded on three different soils (two typical Natracual's halophyte steppes adjacent but different stories in grazing management (continuous/rotational) and Vertic Argiudol a wet meadow mesophytes with rotational grazing). The second evaluated the ability of each soil property. The removal of the seed cover alone significantly promoted germination in seeds with lower storage period. Soil type hasn't affected germination; meanwhile, seedling establishment was significantly reduced on Natracualf under continuous grazing. Results suggest that effectively $C$. berroi' seminal covers induce dormancy for a few months $(<8)$. Rotational grazing would promote preservation of $C$. berroi in halophyte communities and their absence in vegetal communities corresponding Argiudol vertic soil, would not be for edaphic issues.
\end{abstract}

[Keywords: grassland, alkaline lowlands, regeneration, native species, type of grazing]

\section{INTRODUCCIÓN}

En la Pampa Deprimida la cría de ganado bovino ha sido la actividad pecuaria predominante desde el siglo XIX, siendo desde entonces el pastizal el principal sustento forrajero y proveedor de servicios (Soriano 1991). En la actualidad, se estima que el pastizal ocupa un $75 \%$ de la superficie regional. Formado por especies con síndrome

victorarielbolaos@gmail.com fotosintético $\mathrm{C}_{3} \mathrm{y} \mathrm{C}_{4}$ que integran mosaicos de comunidades vegetales y distribuyen su producción en el tiempo y en el espacio (Sala et al. 1981; Perelman et al. 2001). La Estepa de halófitas (Perelman et al. 2001; Burkart et al. 2005) cubre alrededor del $16 \%$ de la superficie de la región y ocupa los suelos con altos niveles de salinidad y/o sodicidad en todo su perfil (León et al. 1979; Perelman et al. 2001). La historia pastoril de éste ambiente le 
habría inducido una pérdida en su diversidad florística, promoviendo a Distichlis spicata y $D$. scoparia como especies dominantes (León et al. 1979) y afectado las características físicas y químicas del suelo, reduciendo su contenido de materia orgánica y su tasa de infiltración y aumentando los niveles de alcalinidad y salinidad (Vecchio, 2014). Tales cambios florísticos y edáficos tienen al sobrepastoreo como principal modelador del sistema y cuyas consecuencias se reflejan al mediano plazo en una baja productividad primaria neta y de pobre calidad forrajera (Hidalgo et al. 1998).

La recuperación y el mantenimiento de las especies nativas de alto valor forrajero, adaptadas a las condiciones edafoclimáticas del lugar, constituye una alternativa de regeneración forrajera del ecosistema para aquellos ambientes degradados por el sobrepastoreo. Para llevar adelante dicho objetivo han sido evaluadas distintas estrategias como la aplicación de prácticas que promueven el pulso germinativo del banco de semilla del suelo o la incorporación de semillas de gramíneas nativas (Quiroga et al. 2009). Dichas alternativas denotan ventajas respecto a las estrategias convencionales de reemplazo de la comunidad natural por pasturas monofíticas perennes, generalmente con especies subtropicales de buena productividad y moderado valor forrajero. Entre esas ventajas se destaca la posibilidad de conservar la heterogeneidad y estabilidad del sistema, y respetar la fragilidad estructural y funcional de la comunidad original. Sin embargo, la posibilidad de restaurar los terrenos degradados utilizando especies nativas, requiere un conocimiento profundo de los aspectos biológicos y ecológicos básicos de las especies a tratar (Mollard 2007). Aspectos que en la actualidad son desconocidos para la mayoría de las especies nativas de la Estepa de halófitas del pastizal natural de la Pampa Deprimida.

La germinación es un aspecto central de la biología de las especies y resulta de vital importancia para el mantenimiento y recuperación de sus poblaciones (Rees 1997). Para que una semilla germine requiere el cumplimento de un determinado conjunto de condiciones externas e internas (Rees 1997; Probert 2000). Puede ocurrir, que ante condiciones externas óptimas, la semilla no germine por efecto de dormición (Baskin \& Baskin 1998). Este es un fenómeno usual en semillas no domesticadas (Shaidaeeet al. 1969), más en los pastos de verano (Voigtet al. 1987) y reconocido en algunas especies del pastizal de la Pampa Deprimida (Ahringet al. 1975; Schrauf 1998; Ferrari 1999, 2002). Identificar dicho fenómeno y descifrar los mecanismos responsables a fin de poder prever pretratamientos de la semilla que permitan aumentos en el logro germinativo y conocer el comportamiento de la germinación y establecimiento de la especie sobre los suelos en los sitios a mejorar, es una información crucial para aplicar estrategias alternativas de regeneración forrajera en ambientes degradados por el sobrepastoreo.

En Setaria lachnea, se ha documentado la presencia de inhibidores en las estructuras que recubren las cariópsides, como principal determinante de su dormición (Schrauf 1998). En especies del género Bothriochloa, la remoción manual de las estructuras externas indujo aumentos en la germinación total (Ahringet al. 1975). También ha sido reportado, para varias especies nativas del pastizal de la Pampa Deprimida, como un determinado tiempo de almacenamiento de las semillas basto para atenuar en gran medida el fenómeno de su dormición (Ferrari 1999, 2002). En tanto a lo referido al comportamiento germinativo y capacidad de establecimiento de las especies nativas sobre los distintos tipos de suelos, o en un mismo suelo pero con propiedades físico químicas distintas (producto de la historia pastoril), no ha sido hasta la actualidad evaluado.

Chloris berroi es una gramínea de verano de buen valor forrajero, nativa de los pastizales del norte de La Pampa Deprimida y tiene la particularidad de encontrarse casi exclusivamente en los ambientes halomórficos (León et al. 1979). Se desconoce cuáles son los mecanismosquedesencadenansugerminación y establecimiento en dichos ambientes. El objetivo general de este trabajo es contribuir al aporte de información referente a los aspectos biológicos y ecológicos vinculados a la germinación y establecimiento de $C$. berroi. Se espera además que los resultados contribuyan a explicar la distribución diferencial de la especie en los ambientes halomórficos del pastizal y bajo diferentes historias pastoriles. Las hipótesis planteadas son: 1) La dormición de C. berroi se debe a las estructuras que recubren la cariópside e inhiben su germinación durante cierto lapso de tiempo luego de la cosecha. 2) C. berroi presenta sensibilidad durante su germinación y establecimiento al tipo de sustrato edáfico. 


\section{MATERIALES y MÉTODOS}

Se realizaron dos ensayos consecutivos. (1) Bajo condiciones controladas de laboratorio se evaluó el efecto que la remoción de glumas y glumelas ejerce sobre la germinación en semillas de C. berroi con dos tiempos de almacenamiento ( 2 y 8 meses) y sembradas sobre tres suelos distintos: dos Natracualf típicos (USDA 2010) correspondientes a dos Estepa de halófitas contiguas pero con diferentes historias en el manejo del pastoreo (continuo y rotativo) durante más de diez años y el tercero un suelo Argiudol Vértico (USDA 2010) sin problemas de alcalinidad ni salinidad tomado de una pradera húmeda de mesófitas (Perelman et al. 2001; Burkart et al. 2005) bajo pastoreo rotativo. (2) Una vez finalizados los ensayos germinativos, desde el día 21 posterior a la siembra y hasta el día 66 , se midió bajo condiciones de invernáculo el efecto que cada uno de los suelos ejerce sobre el establecimiento y desarrollo en plántulas de C. berroi.

Las semillas se colectaron manualmente en el mes de febrero de 2011, en una Estepa de halófitas

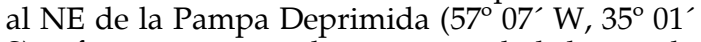
S) y fueron conservadas en oscuridad dentro de bolsas de papel madera $\left(20^{\circ} \mathrm{C} \pm 5{ }^{\circ} \mathrm{C}\right)$. Previo a los tratamientos se efectuaron tres ensayos de viabilidad mediante el test de Tetrazolio y siguiendo diferentes protocolos (ISTA 2013), pero los resultados no fueron congruentes con lo observado en los ensayos germinativos (se obtuvieron valores de viabilidad inferiores al poder germinativo). Esto es común en semillas de especies nativas para las cuales no existe protocolo específico del test de Tetrazolio. Debido a esto, se decidió utilizar semillas potencialmente viables, asumiendo que, en caso de existir, la proporción de semillas no viables no debería diferir entre los tratamientos. Para ello, se sembraron únicamente semillas previamente seleccionadas por brillo y turgencia, rasgos externos característico de semillas viables (Baskin \& Baskin 1998). Para el pretratamiento "pelado" se removieron las estructuras que recubren la cariópside (glumas y glumelas), de forma manual con pinza y bisturí y bajo lupa binocular, seleccionando las potencialmente viables. En el caso de las semillas "no peladas", las semillas potencialmente viables no pudieron ser seleccionadas de forma directa (lo que se sembró fue la espiguilla). Por ello se debió hacer una estimación del equivalente de espiguillas que resultase igual al número de semillas con buen brillo y turgencia. Para ello, en cada siembra, se tomó una población muestra de 2.000 espiguillas que resultasen no vanas al tacto (al ser presionadas por la yema del dedo sobre la mesada notase por tacto la presencia de semilla en el interior). A 1000 de ellas, se las observó mediante pelado con pinza y bisturí y bajo lupa binocular para contabilizar la cantidad de semillas con buen brillo y turgencia presente, y a la otra mitad se la utilizó para la siembra. El número de espiguillas puestas a germinar se corrigió por esta equivalencia. Así, se
Tabla 1. Valores de materia orgánica (MO), protones de hidrogeno $(\mathrm{pH})$, conductividad eléctrica (Ce), especie/s predominante del staf (Sps pred), porcentaje de cobertura total para cada uno de los sitios de muestreo.

Table 1. Values of organic matter (MO), hydrogen protons $(\mathrm{Ph})$, electrical conductivity (Ce), prevailing species of staf (Sps pred), total coverage area percentage of each of the samples

\begin{tabular}{|c|c|c|c|}
\hline & \multicolumn{2}{|c|}{ Natracualf típico } & \multirow{2}{*}{$\begin{array}{c}\text { Argiudol vértico } \\
\text { Rotativo }\end{array}$} \\
\hline & Continuo & Rotativo & \\
\hline $\mathrm{MO}(\%)$ & 1.5 & 2.9 & 5.3 \\
\hline $\mathrm{pH}$ & 9.5 & 7.5-8 & 6.2 \\
\hline $\mathrm{Ce}\left(\mathrm{dS} . \mathrm{m}^{-1)}\right.$ & 2.4 & 1.4 & 0.5 \\
\hline Sps. Pred. & $\begin{array}{c}\text { Distichlis scopar } \\
\text { D. spicata }\end{array}$ & C. berroi & $\begin{array}{l}\text { Stipa charruana, } \\
\text { Lolium multiflorum }\end{array}$ \\
\hline Cob. (\%) & $30-40$ & $60-80$ & $80-95$ \\
\hline
\end{tabular}

asumió que el número de espiguillas a sembrar que equivalga a 50 semillas con buen brillo turgencia fue de 73 y 68 espiguillas para la $1^{\circ}$ y $2^{\circ}$ siembra respectivamente.

Las muestras de suelo se tomaron de los primeros cinco centímetros del perfil y se llevaron a laboratorio para ser sometidas a un tratamiento de esterilización térmica (método Tyndall, metodología del SAMLA, SAGPyA 2004). Las propiedades físico químicas (Tabla 1) fueron analizadas en estudios previos (Vecchio 2014).

En el ensayo 1 se utilizó un diseño experimental factorial completamente aleatorizado con cinco repeticiones. Factores: tipo de suelo, tiempo de almacenamiento de la semilla ( 2 y 8 meses) y el tratamiento (pelado si/no). La unidad experimental fue la bandeja de $15 \times 10.5 \times 4.5 \mathrm{~cm}$ completada hasta un $90 \%$ con el suelo correspondiente, disgregado y sobre el cual se sembro. Las siembras se efectuaron en superficie colocando 50 semillas previamente seleccionadas o su equivalente en espiguillas. Las condiciones de cámara (alternancia 12hs/12hs y $20 / 30{ }^{\circ} \mathrm{C}$, oscuridad/luz respectivamente) fueron seleccionadas teniendo en cuenta estudios previos sobre otras especies de gramíneas forrajeras $\mathrm{C} 4 \mathrm{y}$ un ensayo preliminar realizado sobre la especie en estudio (Bolaños \&Vecchio 2012, Facultad de Ciencias Agrarias y Forestales. com. pública).

Se hicieron relevamientos diarios y hasta el día 21 del número de semillas germinadas (primera visualización de la plúmula). Los datos fueron llevados a porcentaje de semillas germinadas acumulado al día 21. Para el ensayo 2, en una de las siembras, se dejaron 10 bandejas de cada sustrato y se les hizo un raleo manual hasta dejar en cada una de ellas las 7 mejores plantulitas (densidad equivalente a $120 \mathrm{pl} / \mathrm{m}^{2}$ ). Ya en el invernáculo se mantuvieron bajo condiciones semicontroladas y hasta el día 66. Allí se censó en cada bandeja el número de plántulas establecidas de las 7 seleccionadas, la altura de cada plántula, el número de macollos/plántula, el número de hoja/plántula y el peso (mg de biomasa seca) por plántula. Los 
porcentajes del ensayo 1 y 2 fueron transformados a trasformación angular (arco seno \%) y sometidos a análisis de la varianza. Para las comparaciones de medias se utilizó el test de Tukey $(\mathrm{p}<0.05)$

\section{Resultados}

La remoción de la cubierta seminal promovió significativamente la germinación solo en las semillas de menor tiempo de almacenamiento, mientras que no afectó la germinación de las semillas que recibieron almacenamiento prolongado (Figura 1). Estos efectos interactivos de remoción de cubierta y de tiempo de almacenamiento no fueron afectados por el tipo de sustrato (Tabla 2). Hubo diferencias significativas

Tabla 2. Valores de p y F del análisis de la varianza para: tratamiento pregerminativo de pelado, tiempo de almacenaiento, tipo de suelo y sus la interacciónes.

Table 2. $\mathrm{p}$ y F values of the analysis of variance for: pregerminative treatment of peeling, period elapsed from harvest to sowing and soil type and interaction between them.

\begin{tabular}{lcc}
\hline F.V. & $\mathrm{F}$ & $\mathrm{p}$-valor \\
\hline Pelado & 82.19 & $<0.0001$ \\
Tiempo de almacenamiento & 535.27 & $<0.0001$ \\
suelo & 0.32 & 0.8083 \\
Pelado*tiempo de almacenamiento & 98.73 & $<0.0001$ \\
Pelado*suelo $^{*}$ & 0.73 & 0.5377 \\
Tiempo de almacenamiento*suelo & 3.55 & 0.0192 \\
Pelado*tiempo de almnto*suelo & 0.48 & 0.6988 \\
\hline
\end{tabular}

Tabla 3. Valores medios \pm errores estándar de número de plántulas establecidas/bandeja, número de macollos / planta, número de hojas/planta, altura de planta $(\mathrm{cm})$ y biomasa seca/planta al día 66 puestas sobre tres suelos distintos (dos Natracualf típicos de estepa de halófitas contiguas pero con diferentes historias en el manejo del pastoreo (continuo/rotativo) y un Argiudol Vértico de una pradera húmeda de mesófitas con pastoreo rotativo). Letras distintas entre columnas indican diferencias de medias significativas según el test de Tukey $(\mathrm{p}<=0.05)$.

Table3. Mean values \pm standard errors of the number of established seedlings/, number of tillers / plant, number of leaves / plant, plant height $(\mathrm{cm})$ anddry biomass / plant(g) at day 60 placed upon three different soils (two typical Natracuafl's halophyte steppes adjacent but different stories in grazing management (continuous / rotary) and Vertic Argiudol a wet meadow mesophytes with rotational grazing). Different letters between columns indicate significant mean differences according to Tukey's test ( $\mathrm{p}<=0.05)$.

\begin{tabular}{|c|c|c|c|}
\hline & \multicolumn{2}{|c|}{ Natracualf típico } & \multirow{2}{*}{$\begin{array}{c}\text { Argiudol } \\
\text { vértico } \\
\text { Rotativo }\end{array}$} \\
\hline & Continuo & Rotativo & \\
\hline plantas/maceta & $1.3 \pm 0.47 \mathrm{a}$ & $6.5 \pm 0.27 b$ & $7 \pm 0 b$ \\
\hline altura planta & $14.34 \pm 2.9 \mathrm{a}$ & $20.56 \pm 1.36 b$ & $20.63 \pm 0.93 b$ \\
\hline $\begin{array}{l}\text { (cm) } \\
\text { macollos/planta }\end{array}$ & $2.08 \pm 0.42 \mathrm{a}$ & $2.6 \pm 0.19 a$ & $2.2 \pm 0.14 \mathrm{a}$ \\
\hline hojas/planta & $7.94 \pm 1.53 \mathrm{a}$ & $9.19 \pm 0.6 \mathrm{a}$ & $8.01 \pm 0.42 \mathrm{a}$ \\
\hline $\begin{array}{l}\text { biomasa/planta } \\
\text { (gr) }\end{array}$ & $0.03 \pm 0.01 \mathrm{a}$ & $0.04 \pm 0.00 \mathrm{a}$ & $0.04 \pm 0.00 \mathrm{a}$ \\
\hline
\end{tabular}

en la germinación entre semillas peladas pero sembradas tras distinto tiempo de almacenamiento (Figura 1).

El número de plántulas establecidas y su altura fue significativamente mayor en los

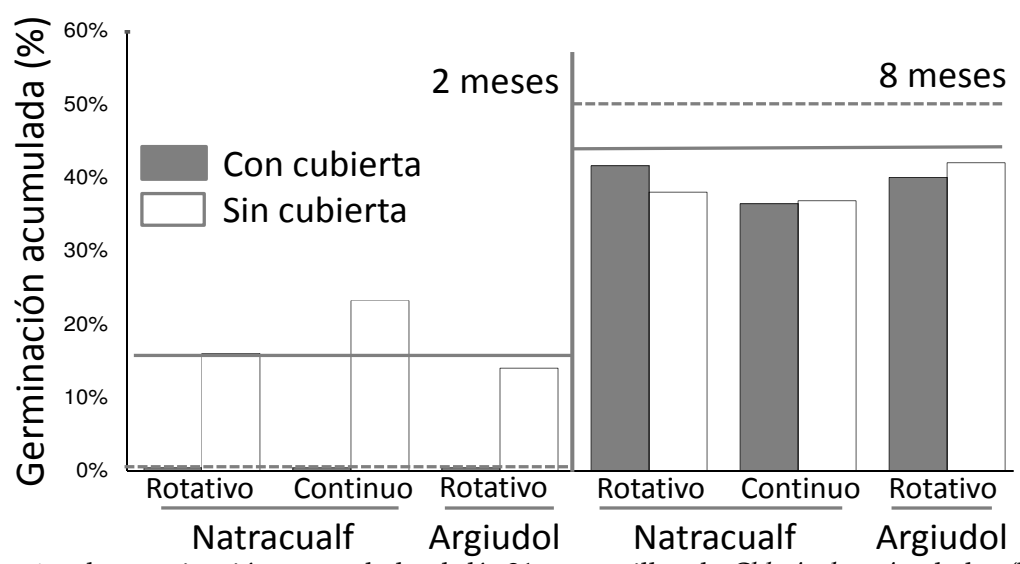

Figura 1. Porcentajes de germinación acumulada al día 21 en semillas de Chloris. berroi peladas (barra bacía) y sin pelar (barra solida) sembrada tras dos tiempos de almacenamiento (2 y 8 meses) y sobre tres suelos distintos (dos Natracualf típicos correspondientes a Estepa de halófitas contiguas pero con diferentes historias en el manejo del pastoreo (continuo/rotativo) durante más de diez años y un Argiudol vértico de una Pradera húmeda de mesófitas bajo pastoreo rotativo) y testigo (algodón) representado con las líneas horizontales en ambas siembras: líneas partidas para semillas no peladas y líneas enteras para semillas peladas.

Figure 1. Accumulated germination (percentage) to day 21th in peeling (empty bars) and unpeeling (full bars) seeds of C. berroi planted after two storing time ( 2 and 8 months) and on top of three different soils (two corresponding to typical Natracualf of adjacent steppe halophytes but with different stories in grazing management (continuous / rotary) for over ten years and a vertic Argiudol of a wet meadow mesophytes under rotational grazing) and the control (cotton) represented with horizontal lines in both planting: broken lines for seed unpeeled and whole line for peeled seed. 
sustratos provenientes de pastoreo rotativo con respecto al encontrado en el sutrato proveniente de pastoreo continuo (Tabla 3). En cambio, no se hallaron diferencias significativas entre los distintos sustratos en el número de macollos/plántula, el número de hojas/plántula y la biomasa seca/plántula. Tampoco hubo diferencias significativas entre suelos distintos (Natracualf tipico/Argiudol vertico) pero con igual historia de manejo del pastoreo (rotativo) (Tabla 3).

\section{DisCUSIÓN}

La dormición juega un papel fundamental en la supervivencia de algunas especies, retardando la germinación hasta el momento en que las condiciones sean las adecuadas para crecer y desarrollarse (Baskin \& Baskin 1998). En la Estepa de halófitas Chloris berroi dispersa sus semillas principalmente a fines de verano-principios de otoño. Nuestros resultados sugieren que las semillas de $C$. berroi presentan un período de dormición menor a 8 meses. Dicho período retrasaría el momento de germinación hasta comenzada la primavera, estación más favorable para el establecimiento de especies estivales en la Pampa Deprimida. Resultado que brinda información a tener en cuenta al planificar el uso pastoril de estos ambientes y más aún, al aplicar técnicas alternativas de regeneración y recuperación forrajera de estos ambientes. Similares resultados fueron encontrados en Briza subaristata (Postulka et al. 2002), Bothriochloa laguroides, Chaetotropis elongata (Ferrari 1999), Sporobolus indicus y Glyceria multiflora (Ferrari 2002), todas forrajeras nativas del pastizal natural de la Pampa Deprimida. En todas ellas un período post cosecha de 6-7 meses fue suficiente para romper, de forma natural, gran parte de la dormición.

El pelado indujo a aumentos significativos en la germinación en semillas con dos meses de almacenamiento. Si bien la magnitud de los porcentajes germinativos obtenidos no podría ser tomada en cuenta para ser evaluada, dado que no fue posible cuantificar el número de semillas viables, esto no impide la evaluación de las diferencias relativas entre tratamientos. Estas diferencias indican que gran parte de la dormición presente en las semillas de $C$. berroi a los dos meses de cosechadas, estaría impuesta por las estructuras que recubren las cariópsides y su remoción previa aminora significativamente dicho fenómeno de forma instantánea. Dando cuenta de, lo altamente eficiente del tratamiento de pelado, al disminuir la dormición en semillas de $C$ berroi sin almacenamiento o con almacenamiento menor a dos meses. Esta respuesta ha sido documentada en otras forrajeras estivales como varias especies del género Bothriochloa (Ahringet al. 1975), Brachiaria dictyoneura (Flores et al. 1997) y Setaria lachnea (Schrauf 1998). Una de las causas del efecto inhibitorio de la germinación por las estructuras que recubren la cariópside es la presencia de sustancias inhibidoras (generalmente hidrófilos y/o termo degradadles) en dichas estructuras (Ahringet al. 1975; Schrauf 1998). En C. berroi el lavado y estratificado no produjo mayor germinación (datos no mostrados). Asimismo, debe tenerse en cuenta que los máximos logros germinativos entre semillas pelada se registraron a los 8 meses de almacenamiento. Esto estaría sugiriendo la presencia de algún proceso de madurez fisiológica post cosecha en la semilla, importante a tener en cuenta.

Los requerimientos edáficos de una especie condicionan el tipo de ambiente donde pueda desarrollarse (Perelman et al. 2001; Burkart et al. 2005). Trabajos recientes demostraron cómo las propiedades físicas y químicas del suelo pueden verse afectadas como consecuencia del pastoreo continúo con niveles de carga animal por encima de los que puede sostener el recurso (Vecchio 2014). C. berroi germinó en todos los sustratos de suelo utilizados, pero en el caso del Natracualf, el pastoreo rotativo sería lo más favorable a sus requerimientos edáficos durante la etapa inicial de su establecimiento. Esto explicaría, en parte, la virtual ausencia de la especie en los stands de comunidades de los bajos alcalinos cuando tiene como dominantes a especies del género Distichlis (León et al. 1979), situación característica de los ambientes donde predomina el Natracualf con comunidades florísticas halomorficas degradadas por efecto del pastoreo continuo. Estos resultados sustentan la conveniencia de implementar el pastoreo rotativo como estrategia de preservación de Chloris berroi. Trabajos recientes demostraron un significativo aumento en la presencia la especie, en los ambientes halomórficos con manejo del pastoreo rotativo frente otros contiguos pero bajo pastoreo continuo (Vecchio 20014).

La distribución natural del género Chloris demuestra una notable adaptación a 
diferentes condiciones de suelos, tanto en especies cultivadas como en especies nativas y naturalizadas (Loch et al. 2004). El crecimiento de las plántulas de $C$. berroi sobre suelos alcalinos (Natracualf típico) fue similar al registrado en el suelo ligeramente ácido de mayor fertilidad (Argiudol vértico). Esto sugiere que la ausencia de la especie en las posiciones más positivas del relieve del pastizal, ambientes más fértiles caracterizados por suelos Argiudoles vérticos, debiera responder a otros factores de tipo no edáficos. Entre dichos factores se destaca su baja habilidad competitiva por factores limitantes en tales condiciones, respecto a las especies dominantes en esos ambientes, hipótesis que debería ser puesta a prueba.

Agradecimientos: especialmente a S. Mejías por su colaboración y acompañamiento en el trabajo de laboratorio y registros, cual hizo posible éste trabajo, a B.Heguy por sus valiosos aportes y a la cátedra de Forrajes de la FCA y F. (UNLP).

\section{BibLIOGRAFIA}

Ahring, RM; JD Estin \& CS CARrison. 1975. Seed appendages and germination of two Asiatic Bluestems. J.Agron., 67:321-325.

BASKIN, CC \& JM BASKIN. 1998. Biogeography and Evolution of Dormancy and Germination. Seed. Ecology. San Diego, Academic Press. pp. 666.

Burkart, SE; MF GARBUlsky; CM GHeRsa; JP Guerschman; JCR LEÓN ET AL. 2005. Las comunidades potenciales del pastizal pampeano bonaerense. En: M OESTERHELD; M Aguiar; C GHersa y J PARUelo (eds). La Heterogeneidad de la Vegetación de los Agroecosistemas. Un Homenaje a Rolando León. Edl. de la Fac. de Agron, UBA.

FERRARI, L. 2002. Condiciones de germinación de semillas de Briza subaristata Lam. recolectadas en diferentes sitios de la Pampa Deprimida (Argentina). Rev. Inv Agr.: Prod y Prot Veg., 17:157-161.

Ferrari, L. 1999. Efecto de la temperatura y de pretratamiento en la germinación de Bothriochloa laguroides (DC.) Herter y Chaetotropis elongata (Kunth) Björkman. Rev. Bras. de Sem., 21:84-87.

Flores, ZV; J Montes \& M Manzano. 1997. Efecto del almacenamiento y tratamiento con ácido sulfurico en semillas de Brachiaria dictyoneura. Zoot. Trop., 16:277-286

Henderson, CB; KE Petersen \& RA Redak, 1988. Spatial and temporal in the seed bank and vegetation of a desert grassland community. J of Ecol., 76:717-728.

Hidalgo, LG; MA Cauhepé M.A. \& AN Erni. 1998. Digestibilidad y contenido de proteína bruta en especies de pastizal de La Pampa Deprimida (Argentina). Rev. de Inv. Agr: Prod. y San Anim., 13:165-177.

LeÓN, RJC; S BuRKART \& CP Movia. 1979. Relevamiento fitosociológico del pastizal del Norte de la Depresión del Salado. Serie Fitogeográfica 17:90 pp. INTA Buenos Aires.

Loch, DS; N Rethman; Van Niekerk, A Willem. 2004. Rhodesgrass. Warm-season C4 grasses, Agronomy monograph., 45:833-872.

MolLARD, FPO. 2007. Regeneración de pastizales naturales por germinación de semillas: control del estado de dormición de las semillas por el contenido hídrico del suelo. Tesis doctoral. Escuela para Graduados Alberto Soriano FAUBA pp. 108.

Perelman, SB; RJC León \& M Oesterheld. 2001 Cross-scale vegetation patterns of Flooding Pampa grasslands. J. Ecol., 89:562-577.

Postulka, EB; C Lopez \& L Ferrari. 2002. Condiciones de germinación de semillas de "Briza subaristata" Lam. recolectadas en diferentes sitios de la Pampa Deprimida (Argentina). Inv. Agr. Prod. y Prot. Veg., 17:157-162

Probert, R. 2000. The role of temperature in germination ecophysiology. En: FENNER, M. (Ed.) Seeds: the Ecology of Regeneration in Plant Communities. CAB International, pp. 261-292.

Quiroga, E; L Blanco y E Orionte. 2009. Evaluación de estrategias de rehabilitación de pastizales áridos. Agosto de 2009. Ecol. Aust, 19:107-117.

Rees, M. 1997. Seed dormancy. En: Crawley, M. (ed). Plant Ecology. Blackwell Science, London, pp. 214-238.

SAGPyA. 2004. (SAMLA). CD-room. ISBN 987-918440-8.

Sala, OE; A Soriano \& S Perelman. 1981. Relaciones hídricas de algunos componentes de un pastizal de la Depresión del Salado. Rev. de la Fac. de Agron., 2:1-10.

SCHRAUF, EG. 1998. Efectos genéticos y ambientales sobre el comportamiento germinativo de Moha perenne. Ecol. Aus., 8:49-56.

Shaedaee, G; BE Dahl \& RM Hansen. 1969 Germination and emergence of different age seed of six grasses. J. of Ran.Manag. Denver, 22:240-245.

Soriano, A. 1991. Río de la Plata Grasslands. Cap. 19 Pp 367-407 en: RT, Coupland (ed). Ecosystems of the World 8A.Natural Grasslands. Introduction and Western Hemisphere. Elsevier, New York.

USDA. 2010. Claves para la Taxonomía de Suelos. Onceava Edición. pp 374.

VECCHIO MC. 2014. Modificaciones en la vegetación y el suelo inducidos por el manejo del pastoreo en la estepa de halófitas de la Pampa Deprimida. Tesis de Magister en Recursos Naturales, Escuela para Graduados Alberto Soriano FAUBA. Argentina. pp. 143.

Voigt, PW; CR Tischler \& MM Povene. 1987. Seed dormancy and its alleviation in lovegrass hybrids. Crop Science, Madison, 36:1699-1705. 